\title{
Proving Properties of Constraint Logic Programs by Eliminating Existential Variables
}

\author{
Alberto Pettorossi ${ }^{1}$, Maurizio Proietti ${ }^{2}$, Valerio Senni ${ }^{1}$ \\ (1) DISP, University of Roma Tor Vergata, Via del Politecnico 1, I-00133 Roma, Italy \\ pettorossi@disp.uniroma2.it, senni@disp.uniroma2.it \\ (2) IASI-CNR, Viale Manzoni 30, I-00185 Roma, Italy \\ proietti@iasi.rm.cnr.it
}

\begin{abstract}
We propose a method for proving first order properties of constraint logic programs which manipulate finite lists of real numbers. Constraints are linear equations and inequations over reals. Our method consists in converting any given first order formula into a stratified constraint logic program and then applying a suitable unfold/fold transformation strategy that preserves the perfect model. Our strategy is based on the elimination of existential variables, that is, variables which occur in the body of a clause and not in its head. Since, in general, the first order properties of the class of programs we consider are undecidable, our strategy is necessarily incomplete. However, experiments show that it is powerful enough to prove several non-trivial program properties.
\end{abstract}

\section{Introduction}

It has been long recognized that program transformation can be used as a means of proving program properties. In particular, it has been shown that unfold/fold transformations introduced in $[4,20]$ can be used to prove several kinds of program properties, such as equivalences of functions defined by recursive equation programs [5,9], equivalences of predicates defined by logic programs [14], first order properties of predicates defined by stratified logic programs [15], and temporal properties of concurrent systems $[7,19]$. In this paper we consider stratified logic programs with constraints and we propose a method based on unfold/fold transformations to prove first order properties of these programs.

The main reason that motivates our method is that transformation techniques may serve as a way of eliminating existential variables (that is, variables which occur in the body of a clause and not in its head) and the consequent quantifier elimination can be exploited to prove first order formulas. Quantifier elimination is a well established technique for theorem proving in first order logic [18] and one of its applications is Tarski's decision procedure for the theory of the field of reals. However, no quantifier elimination method has been developed so far to prove formulas within theories defined by constraint logic programs, where the constraints are themselves formulas of the theory of reals. 
Consider, for instance, the following constraint logic program which defines the membership relation for finite lists of reals:

$$
\begin{aligned}
\text { Member: } & \text { member }(X,[Y \mid L]) \leftarrow X=Y \\
& \text { member }(X,[Y \mid L]) \leftarrow \text { member }(X, L)
\end{aligned}
$$

Suppose we want to show that every finite list of reals has an upper bound, i.e.,

$$
\varphi: \quad \forall L \exists U \forall X(\text { member }(X, L) \rightarrow X \leq U)
$$

Tarski's quantifier elimination method cannot help in this case, because the membership relation is not defined in the language of the theory of reals. The transformational technique we propose in this paper, proves the formula $\varphi$ in two steps. In the first step we transform $\varphi$ into clause form by applying a variant of the Lloyd-Topor transformation [11], thereby deriving the following clauses:

Prop $_{1}$ : 1. prop $\leftarrow \neg p$

2. $p \leftarrow \operatorname{list}(L) \wedge \neg q(L)$

3. $q(L) \leftarrow \operatorname{list}(L) \wedge \neg r(L, U)$

4. $r(L, U) \leftarrow X>U \wedge \operatorname{list}(L) \wedge$ member $(X, L)$

where list $(L)$ holds iff $L$ is a finite list of reals. The predicate prop is equivalent to $\varphi$ in the sense that $M($ Member $) \models \varphi$ iff $M\left(\right.$ Member $\cup$ Prop $\left._{1}\right) \models$ prop, where $M(P)$ denotes the perfect model of a stratified constraint logic program $P$. In the second step, we eliminate the existential variables by extending to constraint logic programs the techniques presented in [16] in the case of definite logic programs. For instance, the existential variable $X$ occurring in the body of the above clause 4 , is eliminated by applying the unfolding and folding rules and transforming that clause into the following two clauses: $r([X \mid L], U) \leftarrow X>U \wedge \operatorname{list}(L)$ and $r([X \mid L], U) \leftarrow r(L, U)$. By iterating the transformation process, we eliminate all existential variables and we derive the following program which defines the predicate prop:

$$
\begin{aligned}
\text { Prop }_{2}: & 1 . \quad \text { prop } \leftarrow \neg p \\
& 2^{\prime} . \quad p \leftarrow p_{1} \\
& 3^{\prime} . \quad p_{1} \leftarrow p_{1}
\end{aligned}
$$

Now, Prop $_{2}$ is a propositional program and has a finite perfect model, which is $\{$ prop $\}$. Since all transformations we have made can be shown to preserve the perfect model, we have that $M($ Member $) \models \varphi$ iff $M\left(\right.$ Prop $\left._{2}\right) \models$ prop and, thus, we have completed the proof of $\varphi$.

The main contribution of this paper is the proposal of a proof method for showing that a closed first order formula $\varphi$ holds in the perfect model of a stratified constraint logic program $P$, that is, $M(P) \models \varphi$. Our proof method is based on program transformations which eliminate existential variables.

The paper is organized as follows. In Section 2 we consider a class of constraint logic programs, called lr-programs ( $l r$ stands for lists of reals), which is Turing complete and for which our proof method is fully automatic. Those programs manipulate finite lists of reals with constraints which are linear equations and inequations over reals. In Section 3 we present the transformation strategy which defines our proof method and we prove its soundness. Due to the undecidability of the first order properties of $l r$-programs, our proof method is 
necessarily incomplete. Some experimental results obtained by using a prototype implementation are presented in Section 5. Finally, in Section 6 we discuss related work in the field of program transformation and theorem proving.

\section{Constraint Logic Programs over Lists of Reals}

We assume that the reals are defined by the usual structure $\mathcal{R}=\langle R, 0,1,+, \cdot, \leq\rangle$. In order to specify programs and formulas, we use a typed first order language [11] with two types: (i) real, denoting the set of reals, and (ii) list of reals (or list, for short), denoting the set of finite lists of reals.

We assume that every element of $R$ is a constant of type real. A term $p$ of type real is defined as follows:

$$
p::=a|X| p_{1}+p_{2} \mid a \cdot X
$$

where $a$ is a real number and $X$ is a variable of type real. We also write $a X$, instead of $a \cdot X$. A term of type real will also be called a linear polynomial. An atomic constraint is a formula of the form: $p_{1}=p_{2}$, or $p_{1}<p_{2}$, or $p_{1} \leq p_{2}$, where $p_{1}$ and $p_{2}$ are linear polynomials. We also write $p_{1}>p_{2}$ and $p_{1} \geq p_{2}$, instead of $p_{2}<p_{1}$ and $p_{2} \leq p_{1}$, respectively. A constraint is a finite conjunction of atomic constraints. A first order formula over reals is a first order formula constructed out of atomic constraints by using the usual connectives and quantifiers (i.e., $\neg, \wedge, \vee, \rightarrow, \exists, \forall)$. By $F_{\mathcal{R}}$ we will denote the set of first order formulas over reals. A term $l$ of type list is defined as follows:

$$
l::=L|[]|[p \mid l]
$$

where $L$ is a variable of type list and $p$ is a linear polynomial. A term of type list will also be called a list. An atom is a formula of the form $r\left(t_{1}, \ldots, t_{n}\right)$ where $r$ is an $n$-ary predicate symbol (with $n \geq 0$ and $r \notin\{=,<, \leq\}$ ) and, for $i=1, \ldots, n, t_{i}$ is either a linear polynomial or a list. An atom is linear if each variable occurs in it at most once. A literal is either an atom (i.e., a positive literal) or a negated atom (i.e., a negative literal). A clause $C$ is a formula of the form: $A \leftarrow c \wedge L_{1} \wedge \ldots \wedge L_{m}$, where: (i) $A$ is an atom, (ii) $c$ is a constraint, and (iii) $L_{1}, \ldots, L_{m}$ are literals. $A$ is called the head of the clause, denoted $h d(C)$, and $c \wedge L_{1} \wedge \ldots \wedge L_{m}$ is called the body of the clause, denoted $b d(C)$.

A constraint logic program over lists of reals, or simply a program, is a set of clauses. A program is stratified if no predicate depends negatively on itself [2]. Given a term or a formula $f$, vars $(f)$ denotes the set of variables occurring in $f$. Given a clause $C$, a variable $V$ is said to be an existential variable of $C$ if $V \in \operatorname{vars}(b d(C))-\operatorname{vars}(h d(C))$.

The definition of a predicate $p$ in a program $P$, denoted by $\operatorname{Def}(p, P)$, is the set of the clauses of $P$ whose head predicate is $p$. The extended definition of $p$ in $P$, denoted by $D e f^{*}(p, P)$, is the union of the definition of $p$ and the definitions of all predicates in $P$ on which $p$ depends (positively or negatively). A program is propositional if every predicate occurring in the program is 0-ary. Obviously, if $P$ is a propositional program then, for every predicate $p, M(P) \models p$ is decidable. 
Definition 1 (lr-program). Let $X$ denote a variable of type real, $L$ a variable of type list, $p$ a linear polynomial, $r_{1}$ and $r_{2}$ two predicate symbols, and $c$ a constraint. An lr-clause is a clause defined as follows:

$$
\begin{aligned}
& \text { head term: } \quad h::=X|[]|[X \mid L] \\
& \text { body term: } \quad b::=p \mid L \\
& \text { lr-clause: } \quad C::=r_{1}\left(h_{1}, \ldots, h_{k}\right) \leftarrow c \\
& r_{1}\left(h_{1}, \ldots, h_{k}\right) \leftarrow c \wedge r_{2}\left(b_{1}, \ldots, b_{m}\right) \\
& r_{1}\left(h_{1}, \ldots, h_{k}\right) \leftarrow c \wedge \neg r_{2}\left(b_{1}, \ldots, b_{m}\right)
\end{aligned}
$$

where: (i) $\operatorname{vars}(p) \neq \emptyset$, (ii) $r_{1}\left(h_{1}, \ldots, h_{k}\right)$ is a linear atom, and (iii) clause $C$ has no existential variables. An lr-program is a finite set of $l r$-clauses.

We assume that the following $l r$-clauses belong to every $l r$-program (but we will omit them when writing $l r$-programs):

$$
\begin{aligned}
& \operatorname{list}([]] \leftarrow \\
& \operatorname{list}([X \mid L]) \leftarrow \operatorname{list}(L)
\end{aligned}
$$

The specific syntactic form of $l r$-programs is required for the automation of the transformation strategy we will introduce in Section 3. Here is an $l r$-program:

$$
\begin{aligned}
P_{1}: & \text { sumlist }([], Y) \leftarrow Y=0 \\
& \text { sumlist }([X \mid L], Y) \leftarrow \operatorname{sumlist}(L, Y-X) \\
& \text { haspositive }([X \mid L]) \leftarrow X>0 \\
& \text { haspositive }([X \mid L]) \leftarrow \text { haspositive }(L)
\end{aligned}
$$

The following definition introduces the class of programs and formulas which can be given in input to our proof method.

Definition 2 (Admissible Pair). Let $P$ be an $l r$-program and $\varphi$ a closed first order formula with no other connectives and quantifiers besides $\neg, \wedge$, and $\exists$. We say that $\langle P, \varphi\rangle$ is an admissible pair if: (i) every predicate symbol occurring in $\varphi$ and different from $\leq,<,=$, also occurs in $P$, (ii) every predicate of arity $n(>0)$ occurring in $P$ and different from $\leq,<,=$, has at least one argument of type list, and (iii) for every proper subformula $\sigma$ of $\varphi$, if $\sigma$ is of the form $\neg \psi$, then either $\sigma$ is a formula in $F_{\mathcal{R}}$ or $\sigma$ has a free occurrence of a variable of type list.

Conditions (ii) and (iii) of Definition 2 are needed to guarantee the soundness of our proof method (see Theorem 3).

Example 1. Let us consider the above program $P_{1}$ defining the predicates sumlist and haspositive, and the formula

$$
\pi: \quad \forall L \forall Y((\operatorname{sumlist}(L, Y) \wedge Y>0) \rightarrow \text { haspositive }(L))
$$

which expresses the fact that if the sum of the elements of a list is positive then the list has at least one positive member. This formula can be rewritten as:

$$
\pi_{1}: \neg \exists L \exists Y(\operatorname{sumlist}(L, Y) \wedge Y>0 \wedge \neg \text { haspositive }(L))
$$

The pair $\left\langle P_{1}, \pi_{1}\right\rangle$ is admissible. Indeed, the only proper subformula of $\pi_{1}$ of the form $\neg \psi$ is $\neg$ haspositive $(L)$ and the free variable $L$ is of type list. 
In order to define the semantics of our logic programs we consider $\mathcal{L} \mathcal{R}$-interpretations where: (i) the type real is mapped to the set of reals, (ii) the type list is mapped to the set of lists of reals, and (iii) the symbols $+, \cdot,=,<, \leq$, [ ], and [_l_] are mapped to the usual corresponding operations and relations on reals and lists of reals. The semantics of a stratified logic program $P$ is assumed to be its perfect $\mathcal{L} \mathcal{R}$-model $M(P)$, which is defined similarly to the perfect model of a stratified logic program $[2,12,17]$ by considering $\mathcal{L} \mathcal{R}$-interpretations, instead of Herbrand interpretations. Note that for every formula $\varphi \in F_{\mathcal{R}}$, we have that $\mathcal{R}=\varphi$ iff for any $\mathcal{L R}$-interpretation $\mathcal{I}, \mathcal{I}=\varphi$.

Now we present a transformation, called Clause Form Transformation, that allows us to derive stratified logic programs starting from formulas, called statements, of the form: $A \leftarrow \beta$, where $A$ is an atom and $\beta$ is a typed first order formula. Our transformation is a variant of the transformation proposed by Lloyd and Topor in [11]. When applying the Clause Form Transformation, we will use the following well known property which guarantees that existential quantification and negation can always be eliminated from first order formulas on reals.

Lemma 1 (Variable Elimination). For any formula $\varphi \in F_{\mathcal{R}}$ there exist $n$ ( $\geq 0)$ constraints $c_{1}, \ldots, c_{n}$ such that: (i) $\mathcal{R} \models \forall\left(\varphi \leftrightarrow\left(c_{1} \vee \ldots \vee c_{n}\right)\right)$, and (ii) every variable in vars $\left(c_{1} \vee \ldots \vee c_{n}\right)$ occurs free in $\varphi$.

In what follows we write $C[\gamma]$ to denote a formula where the subformula $\gamma$ occurs as an outermost conjunct, that is, $C[\gamma]=\gamma_{1} \wedge \gamma \wedge \gamma_{2}$ for some (possibly empty) conjunctions $\gamma_{1}$ and $\gamma_{2}$.

\section{Clause Form Transformation.}

Input: A statement $S$ whose body has no other connectives and quantifiers besides $\neg$, $\wedge$, and $\exists$. Output: A set of clauses denoted $C F T(S)$.

(Step A) Starting from $S$, repeatedly apply the following rules A.1-A.5 until a set of clauses is generated.

(A.1) If $\gamma \in F_{\mathcal{R}}$ and $\gamma$ is not a constraint, then replace $A \leftarrow C[\gamma]$ by the $n$ statements $A \leftarrow C\left[c_{1}\right], \ldots, A \leftarrow C\left[c_{n}\right]$, where $c_{1} \vee \ldots \vee c_{n}$, with $n \geq 0$, is a disjunction of constraints which is equivalent to $\gamma$. (The existence of such a disjunction is guaranteed by Lemma 1 above.)

(A.2) If $\gamma \notin F_{\mathcal{R}}$ then replace $A \leftarrow C[\neg \neg \gamma]$ by $A \leftarrow C[\gamma]$.

(A.3) If $\gamma \wedge \delta \notin F_{\mathcal{R}}$ then replace the statement $A \leftarrow C[\neg(\gamma \wedge \delta)]$ by the two statements $A \leftarrow C\left[\neg\right.$ newp $\left.\left(V_{1}, \ldots, V_{k}\right)\right]$ and newp $\left(V_{1}, \ldots, V_{k}\right) \leftarrow \gamma \wedge \delta$, where newp is a new predicate and $V_{1}, \ldots, V_{k}$ are the variables which occur free in $\gamma \wedge \delta$.

(A.4) If $\gamma \notin F_{\mathcal{R}}$ then replace the statement $A \leftarrow C[\neg \exists V \gamma]$ by the two statements $A \leftarrow C\left[\neg\right.$ newp $\left.\left(V_{1}, \ldots, V_{k}\right)\right]$ and newp $\left(V_{1}, \ldots, V_{k}\right) \leftarrow \gamma$, where newp is a new predicate and $V_{1}, \ldots, V_{k}$ are the variables which occur free in $\exists V \gamma$.

(A.5) If $\gamma \notin F_{\mathcal{R}}$ then replace $A \leftarrow C[\exists V \gamma]$ by $A \leftarrow C\left[\gamma\left\{V / V_{1}\right\}\right]$, where $V_{1}$ is a new variable. 
(Step B) For every clause $A \leftarrow c \wedge G$ such that $L_{1}, \ldots, L_{k}$ are the variables of type list occurring in $G$, replace $A \leftarrow c \wedge G$ by $A \leftarrow c \wedge \operatorname{list}\left(L_{1}\right) \wedge \ldots \wedge \operatorname{list}\left(L_{k}\right) \wedge G$.

Example 2. The set CFT $\left(\right.$ prop $\left._{1} \leftarrow \pi_{1}\right)$, where $\pi_{1}$ is the formula given in Example 1, consists of the following two clauses:

$$
\begin{aligned}
& D_{2}: \operatorname{prop}_{1} \leftarrow \neg \text { new }_{1} \\
& D_{1}: \quad \text { new }_{1} \leftarrow Y>0 \wedge \operatorname{list}(L) \wedge \operatorname{sumlist}(L, Y) \wedge \neg \text { haspositive }(L)
\end{aligned}
$$

(The subscripts of the names of these clauses follow the bottom-up order in which they will be processed by the $U F_{l r}$ strategy we will introduce below.)

By construction, we have that if $\langle P, \varphi\rangle$ is an admissible pair and prop is a new predicate symbol, then $P \cup C F T($ prop $\leftarrow \varphi)$ is a stratified program. The Clause Form Transformation is correct with respect to the perfect $\mathcal{L} \mathcal{R}$-model semantics, as stated by the following theorem.

Theorem 1 (Correctness of CFT). Let $\langle P, \varphi\rangle$ be an admissible pair. Then, $M(P) \models \varphi \quad$ iff $M(P \cup C F T($ prop $\leftarrow \varphi)) \models$ prop.

In general, a clause in $C F T($ prop $\leftarrow \varphi)$ is not an $l r$-clause because, indeed, existential variables may occur in its body. The clauses of $C F T($ prop $\leftarrow \varphi)$ are called typed-definitions. They are defined as follows.

Definition 3 (Typed-Definition, Hierarchy). A typed-definition is a clause of the form: $r\left(V_{1}, \ldots, V_{n}\right) \leftarrow c \wedge \operatorname{list}\left(L_{1}\right) \wedge \ldots \wedge \operatorname{list}\left(L_{k}\right) \wedge G$ where: (i) $V_{1}, \ldots, V_{n}$ are distinct variables of type real or list, and (ii) $L_{1}, \ldots, L_{k}$ are the variables of type list that occur in $G$. A sequence $\left\langle D_{1}, \ldots, D_{n}\right\rangle$ of typed-definitions is said to be a hierarchy if for $i=1, \ldots, n$, the predicate of $h d\left(D_{i}\right)$ does not occur in $\left\{b d\left(D_{1}\right), \ldots, b d\left(D_{i}\right)\right\}$.

One can show that given a closed formula $\varphi$, the set $C F T($ prop $\leftarrow \varphi)$ of clauses can be ordered as a hierarchy $\left\langle D_{1}, \ldots, D_{n}\right\rangle$ of typed-definitions such that $\operatorname{Def}\left(\operatorname{prop},\left\{D_{1}, \ldots, D_{n}\right\}\right)=\left\{D_{k}, D_{k+1}, \ldots, D_{n}\right\}$, for some $k$ with $1 \leq k \leq n$.

\section{The Unfold/Fold Proof Method}

In this section we present the transformation strategy, called $U F_{l r}$ (Unfold/Fold strategy for $l r$-programs), which defines our proof method for proving properties of $l r$-programs. Our strategy applies in an automatic way the transformation rules for stratified constraint logic programs presented in [8]. In particular, the $U F_{l r}$ strategy makes use of the definition introduction, (positive and negative) unfolding, (positive) folding, and constraint replacement rules. (These rules extend the ones proposed in $[6,12]$ where the unfolding of a clause with respect to a negative literal is not permitted.)

Given an admissible pair $\langle P, \varphi\rangle$, let us consider the stratified program $P \cup$ $C F T($ prop $\leftarrow \varphi)$. The goal of our $U F_{l r}$ strategy is to derive a program TransfP 
such that $D e f^{*}($ prop, Transf $P)$ is propositional and, thus, $M($ Transf $P) \models$ prop is decidable. We observe that, in order to achieve this goal, it is enough that the derived program TransfP is an $l r$-program, as stated by the following lemma, which follows directly from Definition 1.

Lemma 2. Let $P$ be an lr-program and $p$ be a predicate occurring in $P$. If $p$ is 0 -ary then $\operatorname{Def}^{*}(p, P)$ is a propositional program.

As already said, the clauses in $C F T($ prop $\leftarrow \varphi)$ form a hierarchy $\left\langle D_{1}, \ldots, D_{n}\right\rangle$ of typed-definitions. The $U F_{l r}$ strategy consists in transforming, for $i=1, \ldots, n$, clause $D_{i}$ into a set of $l r$-clauses. The transformation of $D_{i}$ is performed by applying the following three substrategies, in this order: (i) unfold, which unfolds $D_{i}$ with respect to the positive and negative literals occurring in its body, thereby deriving a set $C s$ of clauses, (ii) replace-constraints, which replaces the constraints appearing in the clauses of $C s$ by equivalent ones, thereby deriving a new set $E s$ of clauses, and (iii) define-fold, which introduces a set NewDefs of new typed-definitions (which are not necessarily $l r$-clauses) and folds all clauses in $E s$, thereby deriving a set $F s$ of $l r$-clauses. Then each new definition in NewDefs is transformed by applying the above three substrategies, and the whole $U F_{l r}$ strategy terminates when no new definitions are introduced. The substrategies unfold, replace-constraints, and define-fold will be described in detail below.

The $U F_{l r}$ Transformation Strategy.

Input: An $l r$-program $P$ and a hierarchy $\left\langle D_{1}, \ldots, D_{n}\right\rangle$ of typed-definitions. Output: A set Defs of typed-definitions including $D_{1}, \ldots, D_{n}$, and an $l r$-program Transf $P$ such that $M(P \cup D e f s)=M($ Transf $P)$.

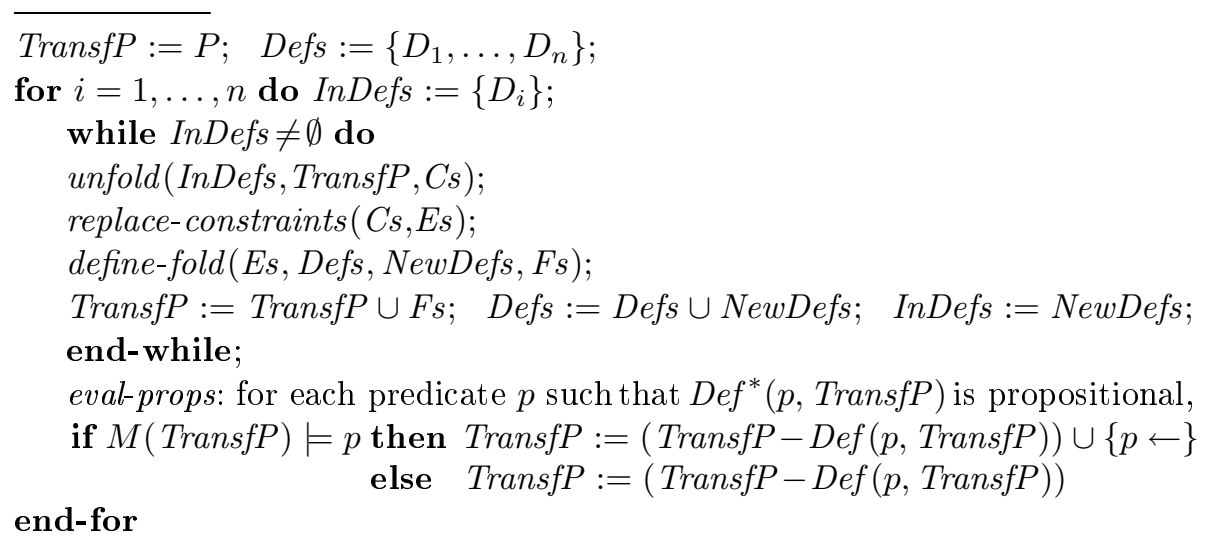

Our assumption that $\left\langle D_{1}, \ldots, D_{n}\right\rangle$ is a hierarchy ensures that, when transforming clause $D_{i}$, for $i=1, \ldots, n$, we only need the clauses obtained after the transformation of $D_{1}, \ldots, D_{i-1}$. These clauses are those of the current value of TransfP.

The following unfold substrategy transforms a set InDefs of typed-definitions by first applying the unfolding rule with respect to each positive literal in the 
body of a clause and then applying the unfolding rule with respect to each negative literal in the body of a clause. In the sequel, we will assume that the conjunction operator $\wedge$ is associative, commutative, idempotent, and with neutral element true. In particular, the order of the conjuncts will not be significant.

The unfold Substrategy.

Input: An lr-program Prog and a set InDefs of typed-definitions.

Output: A set $C s$ of clauses.

Initially, no literal in the body of a clause of InDefs is marked as 'unfolded'.

Positive Unfolding: while there exists a clause $C$ in InDefs of the form $H \leftarrow c \wedge G_{L} \wedge A \wedge G_{R}$, where $A$ is an atom which is not marked as 'unfolded' do Let $C_{1}: K_{1} \leftarrow c_{1} \wedge B_{1}, \ldots, C_{m}: K_{m} \leftarrow c_{m} \wedge B_{m}$ be all clauses of program Prog (where we assume vars $(\operatorname{Prog}) \cap \operatorname{vars}(C)=\emptyset$ ) such that, for $i=1, \ldots, m$, (i) there exists a most general unifier $\vartheta_{i}$ of $A$ and $K_{i}$, and (ii) the constraint $\left(c \wedge c_{i}\right) \vartheta_{i}$ is satisfiable. Let $U$ be the following set of clauses:

$$
U=\left\{\left(H \leftarrow c \wedge c_{1} \wedge G_{L} \wedge B_{1} \wedge G_{R}\right) \vartheta_{1}, \ldots,\left(H \leftarrow c \wedge c_{m} \wedge G_{L} \wedge B_{m} \wedge G_{R}\right) \vartheta_{m}\right\}
$$

Let $W$ be the set of clauses derived from $U$ by removing all clauses of the form

$$
H \leftarrow c \wedge G_{L} \wedge A \wedge \neg A \wedge G_{R}
$$

Inherit the markings of the literals in the body of the clauses of $W$ from those of $C$, and mark as 'unfolded' the literals $B_{1} \vartheta_{1}, \ldots, B_{m} \vartheta_{m}$;

InDefs $:=($ InDefs $-\{C\}) \cup W$;

end-while;

Negative Unfolding: while there exists a clause $C$ in InDefs of the form $H \leftarrow c \wedge G_{L} \wedge \neg A \wedge G_{R}$, where $\neg A$ is a literal which is not marked as 'unfolded' do Let $C_{1}: K_{1} \leftarrow c_{1} \wedge B_{1}, \ldots, C_{m}: K_{m} \leftarrow c_{m} \wedge B_{m}$ be all clauses of program Prog (where we assume that $\operatorname{vars}(\operatorname{Prog}) \cap \operatorname{vars}(C)=\emptyset$ ) such that, for $i=1, \ldots, m$, there exists a most general unifier $\vartheta_{i}$ of $A$ and $K_{i}$. By our assumptions on Prog and on the initial value of InDefs, and as a result of the previous Positive Unfolding phase, we have that, for $i=1, \ldots, m, B_{i}$ is either the empty conjunction true or a literal and $A=K_{i} \vartheta_{i}$. Let $U$ be the following set of statements:

$U=\left\{H \leftarrow c \wedge d_{1} \vartheta_{1} \wedge \ldots \wedge d_{m} \vartheta_{m} \wedge G_{L} \wedge L_{1} \vartheta_{1} \wedge \ldots \wedge L_{m} \vartheta_{m} \wedge G_{R} \mid\right.$

(i) for $i=1, \ldots, m$, either $\left(d_{i}=c_{i}\right.$ and $\left.L_{i}=\neg B_{i}\right)$ or $\left(d_{i}=\neg c_{i}\right.$ and $L_{i}=$ true $)$, and (ii) $c \wedge d_{1} \vartheta_{1} \wedge \ldots \wedge d_{m} \vartheta_{m}$ is satisfiable $\}$

Let $W$ be the set of clauses derived from $U$ by applying as long as possible the following rules:

- remove $H \leftarrow c \wedge G_{L} \wedge \neg$ true $\wedge G_{R}$ and $H \leftarrow c \wedge G_{L} \wedge A \wedge \neg A \wedge G_{R}$

- replace $\neg \neg A$ by $A, \neg\left(p_{1} \leq p_{2}\right)$ by $p_{2}<p_{1}$, and $\neg\left(p_{1}<p_{2}\right)$ by $p_{2} \leq p_{1}$

- replace $H \leftarrow c_{1} \wedge \neg\left(p_{1}=p_{2}\right) \wedge c_{2} \wedge G$ by $H \leftarrow c_{1} \wedge p_{1}<p_{2} \wedge c_{2} \wedge G$

$H \leftarrow c_{1} \wedge p_{2}<p_{1} \wedge c_{2} \wedge G$

Inherit the markings of the literals in the body of the clauses of $W$ from those of $C$, and mark as 'unfolded' the literals $L_{1} \vartheta_{1}, \ldots, L_{m} \vartheta_{m}$;

InDefs $:=($ InDefs $-\{C\}) \cup W$; 
end-while;

$C s:=$ InDefs.

Negative Unfolding is best explained through an example. Let us consider a program consisting of the clauses $C: H \leftarrow c \wedge \neg A, A \leftarrow c_{1} \wedge B_{1}$, and $A \leftarrow c_{2} \wedge B_{2}$. The negative unfolding of $C$ w.r.t. $\neg A$ gives us the following four clauses:

$$
\begin{aligned}
& H \leftarrow c \wedge \neg c_{1} \wedge \neg c_{2} \\
& H \leftarrow c \wedge c_{1} \wedge \neg c_{2} \wedge \neg B_{1} \\
& H \leftarrow c \wedge \neg c_{1} \wedge c_{2} \wedge \neg B_{2} \\
& H \leftarrow c \wedge c_{1} \wedge c_{2} \wedge \neg B_{1} \wedge \neg B_{2}
\end{aligned}
$$

whose conjunction is equivalent to $H \leftarrow c \wedge \neg\left(\left(c_{1} \wedge B_{1}\right) \vee\left(c_{2} \wedge B_{2}\right)\right)$.

Example 3. Let us consider the program-property pair $\left\langle P_{1}, \pi_{1}\right\rangle$ of Example 1. In order to prove that $M\left(P_{1}\right) \models \pi_{1}$, we apply the $U F_{l r}$ strategy starting from the hierarchy $\left\langle D_{1}, D_{2}\right\rangle$ of typed-definitions of Example 2. During the first execution of the body of the for-loop of that strategy, the unfold substrategy is applied, as we now indicate, by using as input the program $P_{1}$ and the set $\left\{D_{1}\right\}$ of clauses. We have the following positive and negative unfolding steps.

Positive Unfolding. By unfolding clause $D_{1}$ w.r.t. list $(L)$ and then unfolding the resulting clauses w.r.t. sumlist $(L, Y)$, we get:

$$
C_{1}: \text { new }_{1} \leftarrow Y>0 \wedge \operatorname{list}(L) \wedge \operatorname{sumlist}(L, Y-X) \wedge \neg \text { haspositive }([X \mid L])
$$

Negative Unfolding. By unfolding clause $C_{1}$ w.r.t. $\neg$ haspositive $([X \mid L])$, we get:

$$
C_{2}: \operatorname{new}_{1} \leftarrow Y>0 \wedge X \leq 0 \wedge \operatorname{list}(L) \wedge \operatorname{sumlist}(L, Y-X) \wedge \neg \text { haspositive }(L)
$$

The correctness of the unfold substrategy follows from the fact that the positive and negative unfoldings are performed according to the rules presented in [8]. The termination of that substrategy is due to the fact that the number of literals which are not marked as 'unfolded' and which occur in the body of a clause, decreases when that clause is unfolded. Thus, we have the following result.

Lemma 3. Let Prog be an lr-program and let InDefs be a set of typed-definitions such that the head predicates of the clauses of InDefs do not occur in Prog. Then, given the inputs Prog and InDefs, the unfold substrategy terminates and returns a set Cs of clauses such that $M($ Prog $\cup$ InDefs $)=M($ Prog $\cup C s)$.

The replace-constraints substrategy derives from a set $C s$ of clauses a new set $E s$ of clauses by applying equivalences between existentially quantified disjunctions of constraints. We use the following two rules: project and clause split.

Given a clause $H \leftarrow c \wedge G$, the project rule eliminates all variables that occur in $c$ and do not occur elsewhere in the clause. Thus, project returns a new clause $H \leftarrow d \wedge G$ such that $\mathcal{R}=\forall\left(\left(\exists X_{1} \ldots \exists X_{k} c\right) \leftrightarrow d\right)$, where: (i) $\left\{X_{1}, \ldots, X_{k}\right\}=$ $\operatorname{vars}(c)-\operatorname{vars}(\{H, G\})$, and (ii) vars $(d) \subseteq \operatorname{vars}(c)-\left\{X_{1}, \ldots, X_{k}\right\}$. In our prototype theorem prover (see Section 5), the project rule is implemented by using a variant of the Fourier-Motzkin Elimination algorithm [1].

The clause split rule replaces a clause $C$ by two clauses $C_{1}$ and $C_{2}$ such that, for $i=1,2$, the number of occurrences of existential variables in $C_{i}$ is less 
than the number of occurrences of existential variables in $C$. The clause split rule applies the following property, which expresses the fact that $\langle\mathcal{R}, \leq\rangle$ is a linear order: $\mathcal{R} \models \forall X \forall Y(X<Y \vee Y \leq X)$. For instance, a clause of the form $H \leftarrow Z \leq X \wedge Z \leq Y \wedge G$, where $Z$ is an existential variable occurring in the conjunction $G$ of literals and $X$ and $Y$ are not existential variables, is replaced by the two clauses $H \leftarrow Z \leq X \wedge X<Y \wedge G$ and $H \leftarrow Z \leq Y \wedge Y \leq X \wedge G$. The decrease of the number of occurrences of existential variables guarantees that we can apply the clause split rule a finite number of times only.

The replace-constraints Substrategy.

Input: A set Cs of clauses. Output: A set Es of clauses.

- Introduce Equations. (A) From $C s$ we derive a new set $R_{1}$ of clauses by applying as long as possible the following two rules, where $p$ denotes a linear polynomial which is not a variable, and $Z$ denotes a fresh new variable:

(R.1) $H \leftarrow c \wedge G_{L} \wedge r(\ldots, p, \ldots) \wedge G_{R}$ is replaced by $H \leftarrow c \wedge Z=p \wedge G_{L} \wedge r(\ldots, Z, \ldots) \wedge G_{R}$

(R.2) $H \leftarrow c \wedge G_{L} \wedge \neg r(\ldots, p, \ldots) \wedge G_{R}$ is replaced by $H \leftarrow c \wedge Z=p \wedge G_{L} \wedge \neg r(\ldots, Z, \ldots) \wedge G_{R}$

(B) From $R_{1}$ we derive a new set $R_{2}$ of clauses by applying to every clause $C$ in $R_{1}$ the following rule. Let $C$ be of the form $H \leftarrow c \wedge G$. Suppose that $\mathcal{R} \models \forall\left(c \leftrightarrow\left(X_{1}=p_{1} \wedge X_{n}=p_{n} \wedge d\right)\right)$, where: (i) $X_{1}, \ldots, X_{n}$ are existential variables of $C$, (ii) $\operatorname{vars}\left(X_{1}=p_{1} \wedge \ldots \wedge X_{n}=p_{n} \wedge d\right) \subseteq \operatorname{vars}(c)$, (iii) $\left\{X_{1}, \ldots, X_{n}\right\} \cap$ $\operatorname{vars}\left(\left\{p_{1}, \ldots, p_{n}, d\right\}\right)=\emptyset$. Then we replace $C$ by $H \leftarrow X_{1}=p_{1} \wedge \ldots \wedge X_{n}=p_{n} \wedge d \wedge G$.

- Project. We derive a new set $R_{3}$ of clauses by applying to every clause in $R_{2}$ the project rule.

- Clause Split. From $R_{3}$ we derive a new set $R_{4}$ of clauses by applying as long as possible the following rule. Let $C$ be a clause of the form $H \leftarrow c_{1} \wedge c_{2} \wedge c \wedge G$ (modulo commutativity of $\wedge$ ). Let $E$ be the set of existential variables of $C$. Let $X \in E$ and let $d_{1}$ and $d_{2}$ be two inequations such that $\mathcal{R} \models \forall\left(\left(c_{1} \wedge c_{2}\right) \leftrightarrow\right.$ $\left.\left(d_{1} \wedge d_{2}\right)\right)$. Suppose that: (i) $d_{1} \wedge d_{2}$ is of one of the following six forms:

$$
\begin{array}{lll}
X \leq p_{1} \wedge X \leq p_{2} & X \leq p_{1} \wedge X<p_{2} & X<p_{1} \wedge X<p_{2} \\
p_{1} \leq X \wedge p_{2} \leq X & p_{1} \leq X \wedge p_{2}<X & p_{1}<X \wedge p_{2}<X
\end{array}
$$

and (ii) $\left(\operatorname{vars}\left(p_{1}\right) \cup \operatorname{vars}\left(p_{2}\right)\right) \cap E=\emptyset$.

Then $C$ is replaced by the following two clauses: $C_{1}: H \leftarrow d_{1} \wedge p_{1}<p_{2} \wedge c \wedge G$ and $C_{2}: H \leftarrow d_{2} \wedge p_{2} \leq p_{1} \wedge c \wedge G$, and then each clause in $\left\{C_{1}, C_{2}\right\}$ with an unsatisfiable constraint in its body is removed.

- Eliminate Equations. From $R_{4}$ we derive the new set $E s$ of clauses by applying to every clause $C$ in $R_{4}$ the following rule. If $C$ is of the form $H \leftarrow X_{1}=p_{1}$ $\wedge \ldots \wedge X_{n}=p_{n} \wedge d \wedge G$ where $\left\{X_{1}, \ldots, X_{n}\right\} \cap \operatorname{vars}\left(\left\{p_{1}, \ldots p_{n}, d\right\}\right)=\emptyset$, then $C$ is replaced by $(H \leftarrow d \wedge G)\left\{X_{1} / p_{1}, \ldots, X_{n} / p_{n}\right\}$. 
The transformation described at Point (A) of Introduce Equations allows us to treat all polynomials occurring in the body of a clause in a uniform way as arguments of constraints. The transformation described at Point (B) of Introduce Equations identifies those existential variables which can be eliminated during the final Eliminate Equations transformation. That elimination is performed by substituting, for $i=1, \ldots, n$, the variable $X_{i}$ by the polynomial $p_{i}$.

Example 4. By applying the replace-constraints substrategy, clause $C_{2}$ of Example 3 is transformed as follows. By introducing equations we get:

$C_{3}:$ new $_{1} \leftarrow Y>0 \wedge X \leq 0 \wedge Z=Y-X \wedge \operatorname{list}(L) \wedge \operatorname{sumlist}(L, Z) \wedge \neg$ haspositive $(L)$

Then, by applying the project transformation, we get:

$C_{4}:$ new $_{1} \leftarrow Z>0 \wedge \operatorname{list}(L) \wedge \operatorname{sumlist}(L, Z) \wedge \neg$ haspositive $(L)$

The correctness of the replace-constraints substrategy is a straightforward consequence of the fact that the Introduce Equations, Project, Clause Split, and Eliminate Equations transformations are performed by using the rule of replacement based on laws presented in [8]. The termination of Introduce Equations and Eliminate Equations is obvious. The termination of Project is based on the termination of the specific algorithm used for variable elimination (e.g., FourierMotzkin algorithm). As already mentioned, the termination of Clause Split is due to the fact that at each application of this transformation the number of occurrences of existential variables decreases. Thus, we get the following lemma.

Lemma 4. For any program Prog and set $C s \subseteq$ Prog of clauses, the replaceconstraints substrategy with input Cs terminates and returns a set Es of clauses such that $M($ Prog $)=M(($ Prog $-C s) \cup E s)$.

The define-fold substrategy eliminates all existential variables in the clauses of the set Es obtained after the unfold and replace-constraints substrategies. This elimination is done by folding all clauses in Es that contain existential variables. In order to make these folding steps we use the typed-definitions in Defs and, if necessary, we introduce new typed-definitions which we add to the set NewDefs.

The define-fold Substrategy.

Input: A set Es of clauses and a set Defs of typed-definitions.

Output: A set NewDefs of typed-definitions and a set Fs of $l r$-clauses.

Initially, both NewDefs and Fs are empty.

for each clause $C: H \leftarrow c \wedge G$ in $E s$ do

if $C$ is an $l r$-clause then $F s:=F s \cup\{C\}$ else

- Define. Let $E$ be the set of existential variables of $C$. We consider a clause NewD of the form newp $\left(V_{1}, \ldots, V_{m}\right) \leftarrow d \wedge B$ constructed as follows:

(1) let $c$ be of the form $c_{1} \wedge c_{2}$, where vars $\left(c_{1}\right) \cap E=\emptyset$ and for every atomic constraint $a$ occurring in $c_{2}$, vars $(a) \cap E \neq \emptyset$; let $d \wedge B$ be the most general (modulo variants) conjunction of constraints and literals such that there exists a substitution $\vartheta$ with the following properties: (i) $(d \wedge B) \vartheta=c_{2} \wedge G$, and (ii) for 
each binding $V / p$ in $\vartheta, V$ is a variable not occurring in $C, \operatorname{vars}(p) \neq \emptyset$, and $\operatorname{vars}(p) \cap E=\emptyset$;

(2) newp is a new predicate symbol;

(3) $\left\{V_{1}, \ldots, V_{m}\right\}=\operatorname{vars}(d \wedge B)-E$.

NewD is added to NewDefs, unless in Defs there exists a typed-definition $D$ which is equal to NewD, modulo the name of the head predicate, the names of variables, equivalence of constraints, and the order and multiplicity of literals in the body. If such a clause $D$ belongs to Defs and no other clause in Defs has the same head predicate as $D$, then we assume that $N e w D=D$.

- Fold. Clause $C$ is folded using clause NewD as follows:

$F s:=F s \cup\left\{H \leftarrow c_{1} \wedge\right.$ newp $\left.\left(V_{1}, \ldots, V_{m}\right) \vartheta\right\}$.

end-for

Example 5. Let us consider the clause $C_{4}$ derived at the end of Example 4 . The Define phase produces a typed-definition which is a variant of the typeddefinition $D_{1}$ introduced at the beginning of the application of the strategy (see Example 2). Thus, $C_{4}$ is folded using clause $D_{1}$, and we get the clause:

$$
C_{5}: \text { new }_{1} \leftarrow \text { new }_{1}
$$

Let us now describe how the proof of $M\left(P_{1}\right) \models \pi_{1}$ proceeds. The program TransfP derived so far consists of clause $C_{5}$ together with the clauses defining the predicates list, sumlist, and haspositive. Thus, Def ${ }^{*}\left(\right.$ new $_{1}$, TransfP $)$ consists of clause $C_{5}$ only, which is propositional and, by eval-props, we remove $C_{5}$ from TransfP because $M$ (Transf $P) \not \models n e w_{1}$. The strategy continues by considering the typed definition $D_{2}$ (see Example 2). By unfolding $D_{2}$ with respect to $\neg n e w_{1}$ we get the final program TransfP, which consists of the clause prop $_{1} \leftarrow$ together with the clauses for list, sumlist, and haspositive. Thus, $M($ TransfP $) \models$ prop $_{1}$ and, therefore, $M\left(P_{1}\right) \models \pi_{1}$.

The proof of correctness for the define-fold substrategy is more complex than the proofs for the other substrategies. The correctness results for the unfold/fold transformations presented in [8] guarantee the correctness of a folding transformation if each typed-definition used for folding is unfolded w.r.t. a positive literal during the application of the $U F_{l r}$ transformation strategy. The fulfillment of this condition is ensured by the following two facts: (1) by the definition of an admissible pair and by the definition of the Clause Form Transformation, each typed-definition has at least one positive literal in its body (indeed, by Condition (iii) of Definition 2 each negative literal in the body of a typed-definition has at least one variable of type list and, therefore, the body of the typed-definition has at least one list atom), and (2) in the Positive Unfolding phase of the unfold substrategy, each typed-definition is unfolded w.r.t. all positive literals.

Note that the set $F s$ of clauses derived by the define-fold substrategy is a set of $l r$-clauses. Indeed, by the unfold and replace-constraints substrategies, we derive a set $E s$ of clauses of the form $r\left(h_{1}, \ldots, h_{k}\right) \leftarrow c \wedge G$, where $h_{1}, \ldots, h_{k}$ are head terms (see Definition 1). By folding we derive clauses of the form

$$
r\left(h_{1}, \ldots, h_{k}\right) \leftarrow c_{1} \wedge \operatorname{newp}\left(V_{1}, \ldots, V_{m}\right) \vartheta
$$


where $\operatorname{vars}\left(c_{1} \wedge \operatorname{newp}\left(V_{1}, \ldots, V_{m}\right) \vartheta\right) \subseteq \operatorname{vars}\left(r\left(h_{1}, \ldots, h_{k}\right)\right)$, and for $i=1, \ldots, m$, $\operatorname{vars}\left(V_{i} \vartheta\right) \neq \emptyset$ (by the conditions at Points (1)-(3) of the Define phase). Hence, all clauses in $F s$ are $l r$-clauses.

The termination of the define-fold substrategy is obvious, as each clause is folded at most once. Thus, we have the following result.

Lemma 5. During the $U F_{l r}$ strategy, if the define-fold substrategy takes as inputs the set Es of clauses and the set Defs of typed-definitions, then this substrategy terminates and returns a set NewDefs of typed-definitions and a set Fs of lr-clauses such that $M($ Transf $P \cup E s \cup N e w D e f s)=M($ Transf $P \cup F s \cup N e w D e f s)$.

By using Lemmata 3, 4, and 5 we get the following correctness result for the $U F_{l r}$ strategy.

Theorem 2. Let $P$ be an lr-program and $\left\langle D_{1}, \ldots, D_{n}\right\rangle$ a hierarchy of typeddefinitions. Suppose that the $U F_{l r}$ strategy with inputs $P$ and $\left\langle D_{1}, \ldots, D_{n}\right\rangle$ terminates and returns a set Defs of typed-definitions and a program TransfP. Then: (i) TransfP is an lr-program and (ii) $M(P \cup D e f s)=M($ Transf $P)$.

Now, we are able to prove the soundness of the unfold/fold proof method.

Theorem 3 (Soundness of the Unfold/Fold Proof Method). Let $\langle P, \varphi\rangle$ be an admissible pair and let $\left\langle D_{1}, \ldots, D_{n}\right\rangle$ be the hierarchy of typed-definitions obtained from prop $\leftarrow \varphi$ by the Clause Form Transformation. If the UF lr strategy with inputs $P$ and $\left\langle D_{1}, \ldots, D_{n}\right\rangle$ terminates and returns a program Transf $P$, then:

$$
M(P) \models \varphi \quad \text { iff } \quad(\text { prop } \leftarrow) \in \text { TransfP }
$$

Proof. By Theorem 1 and Point (ii) of Theorem 2, we have that $M(P) \models \varphi$ iff $M($ TransfP $) \models$ prop. By Point (i) of Theorem 2 and Lemma 2 we have that $D e f^{*}($ prop, Transf $P)$ is propositional. Since the last step of the $U F_{l r}$ strategy is an application of the eval-props transformation, we have that $D e f^{*}$ (prop,TransfP) is either the singleton $\{$ prop $\leftarrow\}$, if $M($ Transf $P) \models$ prop, or the empty set, if $M($ TransfP $) \not \neq$ prop.

\section{A Complete Example}

As an example of application of our transformation strategy for proving properties of constraint logic programs we consider the $l r$-program Member and the property $\varphi$ given in the Introduction. The formula $\varphi$ is rewritten as follows:

$\varphi_{1}: \neg \exists L \neg \exists U \neg \exists X(X>U \wedge \operatorname{member}(X, L))$

The pair $\left\langle\right.$ Member, $\left.\varphi_{1}\right\rangle$ is admissible. By applying the Clause Form Transformation starting from the statement prop $\leftarrow \varphi_{1}$, we get the following clauses:

$D_{4}:$ prop $\leftarrow \neg p$

$D_{3}: p \longleftarrow \operatorname{list}(L) \wedge \neg q(L)$

$D_{2}: q(L) \leftarrow \operatorname{list}(L) \wedge \neg r(L, U)$

$D_{1}: r(L, U) \leftarrow X>U \wedge \operatorname{list}(L) \wedge \operatorname{member}(X, L)$ 
where $\left\langle D_{1}, D_{2}, D_{3}, D_{4}\right\rangle$ is a hierarchy of typed-definitions. Note that the three nested negations in $\varphi_{1}$ generate the three atoms $p, q(L)$, and $r(L, U)$ with their typed-definitions $D_{3}, D_{2}$, and $D_{1}$, respectively. The arguments of $p, q$, and $r$ are the free variables of the corresponding subformulas of $\varphi_{1}$. For instance, $r(L, U)$ corresponds to the subformula $\exists X(X>U \wedge \operatorname{member}(X, L))$ which has $L$ and $U$ as free variables. Now we apply the $U F_{l r}$ strategy starting from the program Member and the hierarchy $\left\langle D_{1}, D_{2}, D_{3}, D_{4}\right\rangle$.

- Execution of the for-loop with $i=1$. We have: InDefs $=\left\{D_{1}\right\}$. By unfolding clause $D_{1}$ w.r.t. the atoms $\operatorname{list}(L)$ and member $(X, L)$ we get:

$1.1 r([X \mid T], U) \leftarrow X>U \wedge \operatorname{list}(T)$

$1.2 r([X \mid T], U) \leftarrow Y>U \wedge \operatorname{list}(T) \wedge \operatorname{member}(Y, T)$

No replacement of constraints is performed. Then, by folding clause 1.2 using $D_{1}$, we get:

$$
1.3 r([X \mid T], U) \leftarrow r(T, U)
$$

After the define-fold substrategy the set $F$ s of clauses is $\{1.1,1.3\}$, and at this point the program TransfP is Member $\cup\{1.1,1.3\}$. No new definitions are introduced and, thus, InDefs $=\emptyset$ and the while-loop terminates. eval-props is not performed because the predicate $r$ is not propositional.

- Execution of the for-loop with $i=2$. We have: InDefs $=\left\{D_{2}\right\}$. We unfold clause $D_{2}$ w.r.t. $\operatorname{list}(L)$ and $\neg r(L, U)$, we get:

$2.1 q([]) \leftarrow$

$2.2 q([X \mid T]) \leftarrow X \leq U \wedge \operatorname{list}(T) \wedge \neg r(T, U)$

No replacement of constraints is performed. Then we introduce the new definition:

$2.3 q_{1}(X, T) \leftarrow X \leq U \wedge \operatorname{list}(T) \wedge \neg r(T, U)$

and we fold clause 2.2 using clause 2.3 . We get:

$2.4 q([X \mid T]) \leftarrow q_{1}(X, T)$

Since NewDefs $=$ InDefs $=\{2.3\}$ we execute again the body of the while-loop. By unfolding clause 2.3 w.r.t. list $(T)$ and $\neg r(T, U)$, we get:

$2.5 q_{1}(X,[]) \leftarrow$

$2.6 q_{1}(X,[Y \mid T]) \leftarrow X \leq U \wedge Y \leq U \wedge \operatorname{list}(T) \wedge \neg r(T, U)$

By applying replace-constraints, clause 2.6 generates the following two clauses:

2.6.1 $q_{1}(X,[Y \mid T]) \leftarrow X>Y \wedge X \leq U \wedge \operatorname{list}(T) \wedge \neg r(T, U)$

2.6.2 $q_{1}(X,[Y \mid T]) \leftarrow X \leq Y \wedge Y \leq U \wedge \operatorname{list}(T) \wedge \neg r(T, U)$

By folding clauses 2.6.1 and 2.6.2 using clause 2.3, we get:

$2.7 q_{1}(X,[Y \mid T]) \leftarrow X>Y \wedge q_{1}(X, T)$

$2.8 q_{1}(X,[Y \mid T]) \leftarrow X \leq Y \wedge q_{1}(Y, T)$

At this point the program TransfP is Member $\cup\{1.1,1.3,2.1,2.4,2.5,2.7,2.8\}$. No new definitions are introduced and, thus, the while-loop terminates. evalprops is not performed because the predicates $q$ and $q_{1}$ are not propositional.

- Execution of the for-loop with $i=3$. We have: InDefs $=\left\{D_{3}\right\}$. By unfolding clause $D_{3}$ w.r.t. $\operatorname{list}(L)$ and $\neg q(L)$, we get: 
$3.1 p \leftarrow \operatorname{list}(T) \wedge \neg q_{1}(X, T)$

No replacement of constraints is performed. The following new definition:

$3.2 p_{1} \leftarrow \operatorname{list}(T) \wedge \neg q_{1}(X, T)$

is introduced. Then by folding clause 3.1 using clause 3.2 , we get:

$3.3 p \leftarrow p_{1}$

Since NewDefs $=$ InDefs $=\{3.2\}$ we execute again the body of the while-loop. By unfolding clause 3.2 w.r.t. $\operatorname{list}(T)$ and $\neg q_{1}(X, T)$, we get:

$3.4 p_{1} \leftarrow X>Y \wedge \operatorname{list}(T) \wedge \neg q_{1}(X, T)$

$3.5 p_{1} \leftarrow X \leq Y \wedge \operatorname{list}(T) \wedge \neg q_{1}(Y, T)$

Since the variable $Y$ occurring in the constraints $X>Y$ and $X \leq Y$ is existential, we apply the project rule to clauses 3.4 and 3.5 and we get the following clause:

$3.6 p_{1} \leftarrow \operatorname{list}(T) \wedge \neg q_{1}(X, T)$

This clause can be folded using clause 3.2 , thereby deriving the following clause:

$3.7 p_{1} \leftarrow p_{1}$

Clauses 3.3 and 3.7 are added to TransfP. Since the predicates $p$ and $p_{1}$ are both propositional, we execute eval-props. We have that: (i) $M$ (TransfP) $\mid \vDash p_{1}$ and (ii) $M$ (TransfP) $\forall p$. Thus, clauses 3.3 and 3.7 are removed from TransfP. Hence, Transf $P=$ Member $\cup\{1.1,1.3,2.1,2.4,2.5,2.7,2.8\}$.

- Execution of the for-loop with $i=4$. We have: InDefs $=\left\{D_{4}\right\}$. By unfolding clause $D_{4}$ w.r.t. $\neg p$, we get the clause:

4. prop $\leftarrow$

This clause shows that, as expected, property $\varphi$ holds for any finite list of reals.

\section{Experimental Results}

We have implemented our proof method by using the MAP transformation system [13] running under SICStus Prolog on a $900 \mathrm{MHz}$ Power PC. Constraint satisfaction and entailment were performed using the clp(r) module of SICStus. Our prototype has automatically proved the properties listed in the following table, where the predicates member, sumlist, and haspositive are defined as shown in Sections 1 and 2, and the other predicates have the following meanings: (i) $\operatorname{ord}(L)$ holds iff $L$ is a list of the form $\left[a_{1}, \ldots, a_{n}\right]$ and for $i=1, \ldots, n-1, a_{i} \leq a_{i+1}$, (ii) $\operatorname{sumzip}(L, M, N)$ holds iff $L, M$, and $N$ are lists of the form $\left[a_{1}, \ldots, a_{n}\right]$, $\left[b_{1}, \ldots, b_{n}\right]$, and $\left[a_{1}+b_{1}, \ldots, a_{n}+b_{n}\right]$, respectively, and (iii) leqlist $(L, M)$ holds iff $L$ and $M$ are lists of the form $\left[a_{1}, \ldots, a_{n}\right]$ and $\left[b_{1}, \ldots, b_{n}\right]$, respectively, and for $i=1, \ldots, n, a_{i} \leq b_{i}$. We do not write here the $l r$-programs which define the predicates $\operatorname{ord}(L), \operatorname{sumzip}(L, M, N)$, and leqlist $(L, M)$.

\begin{tabular}{lr}
\hline Property & Time \\
$\forall L \exists M \forall Y($ member $(Y, L) \rightarrow Y \leq M)$ & $140 \mathrm{~ms}$ \\
$\forall L \forall Y(($ sumlist $(L, Y) \wedge Y>0) \rightarrow$ haspositive $(L))$ & $170 \mathrm{~ms}$ \\
$\forall L \forall Y((\operatorname{sumlist}(L, Y) \wedge Y>0) \rightarrow \exists X($ member $(X, L) \wedge X>0))$ & $160 \mathrm{~ms}$ \\
$\forall L \forall M \forall N((\operatorname{ord}(L) \wedge \operatorname{ord}(M) \wedge$ sumzip $(L, M, N)) \rightarrow \operatorname{ord}(N))$ & $160 \mathrm{~ms}$ \\
$\forall L \forall M(($ leqlist $(L, M) \wedge \operatorname{sumlist}(L, X) \wedge \operatorname{sumlist}(M, Y)) \rightarrow X \leq Y)$ & $50 \mathrm{~ms}$ \\
\hline
\end{tabular}




\section{Related Work and Conclusions}

We have presented a method for proving first order properties of constraint logic programs based on unfold/fold program transformations, and we have shown that the ability of unfold/fold transformations to eliminate existential variables [16] can be turned into a useful theorem proving method. We have provided a fully automatic strategy for the class of $l r$-programs, which are programs acting on reals and finite lists of reals, with constraints as linear equations and inequations over reals. The choice of lists is actually a simplifying assumption we have made and we believe that the extension of our method to any finitely generated data structure is quite straightforward. However, the use of constraints over the reals is an essential feature of our method, because quantifier elimination from constraints is a crucial subprocedure of our transformation strategy.

The first order properties of $l r$-programs are undecidable (and not even semi-decidable), because one can encode every partial recursive function as an $l r$-program without list arguments. As a consequence our proof method is necessarily incomplete. We have implemented the proof method based of program transformation and we have proved some simple, yet non-trivial, properties. As the experiments show, the performance of our method is encouraging.

Our method is an extension of the method presented in [15] which considers logic programs without constraints. The addition of constraints is a very relevant feature, because it provides more expressive power and, as already mentioned, we may use special purpose theorem provers for checking constraint satisfaction and for quantifier elimination. Our method can also be viewed as an extension of other techniques based on unfold/fold transformations for proving equivalences of predicates [14,19], and indeed, our method can deal with a class of first order formulas which properly includes equivalences.

Some papers have proposed transformational techniques to prove propositional temporal properties of finite and/or infinite state systems (see, for instance, $[7,10,19])$. Since propositional temporal logic can be encoded in first order

logic, in principle these techniques can be viewed as instances of the unfold/fold proof method presented here.

However, it should be noted that the techniques described in $[7,10,19]$ have their own peculiarities because they are tailored to the specific problem of verifying concurrent systems.

Finally, we think that a direct comparison of the power of our proof method with that of traditional theorem provers is somewhat inappropriate. The techniques used in those provers are very effective and are the result of a well established line of research (see, for instance, [3] for a survey on the automation of mathematical induction). However, our approach has its novelty and is based on principles which have not been explored in the field of theorem proving. In particular, the idea of making inductive proofs by unfold/fold transformations for eliminating quantifiers, has not yet been investigated within the theorem proving community. 


\section{Acknowledgments}

We would like to thank the anonymous referees for their helpful comments and suggestions.

\section{References}

1. K. R. Apt. Principles of Constraint Programming. Cambridge Univ. Press, 2003.

2. K. R. Apt and R. N. Bol. Logic programming and negation: A survey. Journal of Logic Programming, 19, 20:9-71, 1994.

3. A. Bundy. The automation of proof by mathematical induction. In Handbook of Automated Reasoning, volume I, pages 845-911. North Holland, 2001.

4. R. M. Burstall and J. Darlington. A transformation system for developing recursive programs. Journal of the ACM, 24(1):44-67, January 1977.

5. B. Courcelle. Equivalences and transformations of regular systems - applications to recursive program schemes and grammars. Theor. Comp. Sci., 42:1-122, 1986.

6. S. Etalle and M. Gabbrielli. Transformations of CLP modules. Theoretical Computer Science, 166:101-146, 1996.

7. F. Fioravanti, A. Pettorossi, and M. Proietti. Verifying CTL properties of infinite state systems by specializing constraint logic programs. In Proceedings VCL'01, Florence, Italy, pages 85-96. University of Southampton, UK, 2001.

8. F. Fioravanti, A. Pettorossi, and M. Proietti. Transformation rules for locally stratified constraint logic programs. In Program Development in Computational Logic, LNCS 3049, pages 292-340. Springer, 2004.

9. L. Kott. The McCarthy's induction principle: 'oldy' but 'goody'. Calcolo, 19(1):5969, 1982.

10. M. Leuschel and T. Massart. Infinite state model checking by abstract interpretation and program specialization. In A. Bossi, editor, Proceedings of LOPSTR '99, Venice, Italy, LNCS 1817, pages 63-82. Springer, 1999.

11. J. W. Lloyd. Foundations of Logic Programming. Springer, 1987. 2nd Edition.

12. M. J. Maher. A transformation system for deductive database modules with perfect model semantics. Theoretical Computer Science, 110:377-403, 1993.

13. The MAP System. http://www.iasi.cnr.it/ proietti/system.html.

14. A. Pettorossi and M. Proietti. Synthesis and transformation of logic programs using unfold/fold proofs. Journal of Logic Programming, 41(2\&3):197-230, 1999.

15. A. Pettorossi and M. Proietti. Perfect model checking via unfold/fold transformations. In Proc. CL 2000, London, UK, LNAI 1861, pp. 613-628. Springer, 2000.

16. M. Proietti and A. Pettorossi. Unfolding-definition-folding, in this order, for avoiding unnecessary variables in logic programs. Theor. Comp. Sci., 142(1):89-124, 1995.

17. T. C. Przymusinski. On the declarative semantics of stratified deductive databases and logic programs. In J. Minker, editor, Foundations of Deductive Databases and Logic Programming, pages 193-216. Morgan Kaufmann, 1987.

18. M. O. Rabin. Decidable theories. In Jon Barwise, editor, Handbook of Mathematical Logic, pages 595-629. North-Holland, 1977.

19. A. Roychoudhury, K. Narayan Kumar, C. R. Ramakrishnan, I. V. Ramakrishnan, and S. A. Smolka. Verification of parameterized systems using logic program transformations. In Proc. TACAS 2000, LNCS 1785, pp. 172-187. Springer, 2000.

20. H. Tamaki and T. Sato. Unfold/fold transformation of logic programs. In S.- $\AA$. Tärnlund, ed., Proceedings of ICLP '84, pages 127-138, Uppsala, Sweden, 1984. 